\title{
EFECTO DE PARAMETROS FÍSICOS, QUÍMICO Y SALINIDAD SOBRE LA DENSIDAD POBLACIONAL Y LA COLONIZACIÓN DE MICORRIZAS ARBUSCULARES EN PASTO ANGLETON EN EL MUNICIPIO DE TOLU, SUCRE, COLOMBIA
}

\section{EFFECT OF PHYSICAL, CHEMICAL PARAMETERS AND SALINITY ON THE DENSITY OF POPULATION AND COLONIZATION OF ARBUSCULAR MYCORRHIZAE IN GRASS ANGLETON IN THE MUNICIPALITY OF TOLÚ, SUCRE, COLOMBIA}

PEROZA, C. VICTOR ${ }^{1}$ MsC, PEREZ, C. ALEXANDER ${ }^{2^{*}}$ Dr.

${ }^{1}$ Universidad de Sucre, Facultad de Ciencias Agropecuarias, Colombia. ${ }^{2}$ Universidad de Sucre, Facultad de Ciencias Agropecuarias, Colombia, Grupo de investigación en Bioprospección Agropecuarias.

*Correspondencia alexander.perez@unisucre.edu.co

Recibido: 10-06-2010; Aceptado: 21-07-2010.

\section{Resumen}

El presente estudio tuvo como objetivo determinar el efecto de parámetros físicos, químico y salinidad sobre la densidad poblacional y la colonización de hongos formadores de micorrizas arbusculares (HMA) en pasto angletón en el municipio de Tolú, departamento de Sucre, Colombia. Se seleccionaron aleatoriamente 35 fincas ganaderas sembradas con la especie de pasto angletón, en ellas se tomaron muestras de suelo y de rizosfera a las cuales se realizaron análisis físicoquímico, de salinidad y microbiológico de HMA (número de esporas en $100 \mathrm{~g}$ de suelo y porcentaje de colonización en raíces). Las variables independientes asociadas a parámetros físicos, químico y de salinidad encontradas en el suelo fueron relacionadas con el número de espora y el porcentaje de colonización de HMA mediante análisis estadísticos que permitieran establecer relación directa entre ellas. Fueron encontrados modelos matemáticos, que relacionaron la densidad poblacional de espora de HMA y el porcentaje de colonización en raíces con parámetros físicos-químicos y de salinidad de los suelos. Este es el primer estudio que muestra la relación directa que existe entre parámetros físicos, químicos y de salinidad en función de la esporulación y la colonización de HMA en condiciones de suelos de fincas ganaderas perteneciente al municipio de Tolú en el departamento de Sucre.

Palabras claves: parámetros físico-químicos, salinidad, esporas, colonización.

\section{Abstract}

The objective this study were determine the effect of physical, chemical parameters and salinity on the population density and colonization of arbuscular mycorrhizal fungi (AMF) in grass angleton in the municipality of Tolu, Sucre, Colombia. 35 farms were randomly selected planted with grass species angleton, 
in these samples were taken from soil and rhizosphere of which were carried out physico-chemical, salinity and microbiological AMF (number of spores in $100 \mathrm{~g}$ soil and percentage of root colonization). The independent variables associated with physical, chemical and salinity found in the soil were related to the number of spores and the percentage of AMF colonization by statistical analysis that would allow to establish direct relationship between them. Mathematical models were found that related the density of AMF spores and the percentage of root colonization with physical-chemical parameters and soil salinity. This is the first study to show the direct relationship between physical, chemical and salinity on theof sporulation and colonization of AMF in soil conditions of livestock farms in the municipality of Tolu in the department of Sucre.

Key words: physical-chemical parameters, salinity, spores, colonizatión.

\section{Introducción}

La fuente de alimentación bovina en el departamento es el pastoreo de gramíneas y leguminosas nativas e introducidas, en tal sentido la productividad animal por unidad de superficie está determinada por el valor nutritivo y la producción de forraje que se ofrece (VILORIA, 2002). Entre las especie introducidas el pasto angletón (Dichantium aristatum, Benth) representa la tercera especie con mayor área sembrada en el departamento de Sucre, alcanzando una extensión de aproximadamente 56.200 hectáreas (has), distribuidas en 19 municipios. El municipio de Santiago de Tolú cuya principal actividad económica es la ganadería semi-intensiva cuenta con la segunda mayor área sembrada con esta especie en el departamento, representada en 9.400 (AGUILERA, 2005).

Dado que existe gran diversidad de microorganismos en el ecosistema suelo; los cuales pueden en determinado tiempo y condición, ayudar a la absorción de nutrientes y favorecer a las plantas en situaciones adversas, como es el caso de los hongos formadores de micorrizas arbusculares (HMA), los cuales viviendo en forma simbiótica - mutualista con las plantas cultivadas, ayudan hacer disponibles elementos fijados en el suelo como fósforo, y evita que este sea lavado, además crear tolerancia a estrés hídrico, mejora la agregación de las partículas y ayuda a prevenir la erosión, haciendo posible y rentable la producción vegetal en condiciones desfavorables (JEFFRIES et al., 2003).

La sequía y la salinidad son factores limitantes en la producción agrícola, los estudios sobre micorriza en estos ambientes son relativamente recientes. Diversos autores señalan que la mejora de la nutrición fosforada en plantas micorrizadas es un factor clave que justifica el incremento de tolerancia a condiciones de salinidad (KENNEDY y SMITH, 1995).

Existen escasas investigaciones a nivel mundial y nacional que demuestren 
integración entre los parámetros físicos, químicos y biológicos con relación a la calidad del ecosistema suelo. Las informaciones que existen son pocos conclusivos y de difícil interpretación, dada la gran complejidad del sistema en estudio en donde se evalúan varias características, generando un número mayor de datos, lo que proporcionalmente dificulta su interpretación. Una estrategia para evaluar el conjunto de indicadores biológicos asociados a los ecosistemas comúnmente sugeridos es el análisis a través de herramientas de bioestadística (análisis multivariados) que permita la conversión e interpretación de un conjunto de variables con una alta correspondencia entre ciertos indicadores y un componente, resultando en un alto peso absoluto del indicador en un componente determinado (TOTOLA y CHAER, 2002).

La importancia que tiene la colonización de las HMA con raíces de las plantas, en relación al mejoramiento de las condiciones físico-químicas del suelo; la estimulación del crecimiento e incremento de la calidad nutricional de las especies vegetales, convirtiéndolas en más tolerantes a condiciones adversas tanto abióticas como bióticas. Sin embargo existe poca evidencia in situ, del efecto de estos hongos con las plantas; debido a que en condiciones naturales es difícil de evaluar la relación entre las variables ambientales, esporulación y la colonización de micorrizas arbusculares sobre las plantas.

A partir de la anterior situación, surge la necesidad de evaluar in situ la esporulación y colonización de HMA en función a parámetros físico y químicos del suelo. Estas necesidades justifican la profundización de investigaciones conducentes a determinar el efecto de esos componentes biológicos sobre agroecosistemas de pasturas. El presente trabajo determinó el efecto de parámetros físicos, químicos y salinidad sobre la densidad poblacional y la colonización de micorrizas arbusculares, en pasto angletón en el municipio de Tolú, Sucre, Colombia.

\section{Materiales y métodos}

\section{Localización}

El estudio fue realizado en el municipio de Santiago de Tolú, perteneciente a la subregión llanura costera aluvial o del Golfo del Morrosquillo, ubicado al Noreste del departamento de Sucre, con una extensión de 35750 has con $45 \mathrm{Km}$ de costa, ubicado a una altura que oscila entre 0 y $10 \mathrm{msnm}$. La formación vegetal corresponde al bosque seco tropical (Holdrige), se encuentra en clima cálido seco, los materiales que conforman los suelos están constituidos por sedimentos aluviales, marinos o combinaciones de los dos. En algunos sectores hay 
sedimentos orgánicos (INSTITUTO GEOGRAFICO AGUSTIN CODAZZI, 1999, PLAN DE ORDENAMIENTO TERRITORIAL, 2000).

El muestreo se llevó a cabo en las fincas ganaderas establecidas con el pasto angletón en el municipio de Tolú, en dos zonas agrológicas ubicadas según la clasificación de las tierras por su capacidad de uso en las subclases IIIsc y IVscVhs.

Zona 1 (subclase IIlsc), tierras aptas para la mayoría de los cultivos de clima cálido seco (algodón, arroz, sorgo) su rendimiento aumentará con fertilización y riego, pertenece a esta los siguientes corregimientos, cabecera municipal, Puerto Viejo, Santa Lucia, Nueva Era, Pita en medio y Coveñas.

Zona 2 (subclase IVsc - Vhs), pertenece a esta el corregimiento de Pita Abajo.

Subclase IVsc, tierras aptas para explotación agrícola ayudada con riego y fertilización. Se puede usar en ganadería evitando el sobrepastoreo.

Subclase IVsc - Vhs, en esta asociación la subclase Vhs indica mal drenaje y permeabilidad lenta, condición que se debe mejorar para hacer cultivos.

Subclase Vhs, estas unidades debido a su pobre drenaje son aptas para ganadería en períodos secos y para conservación de fauna.

\section{Muestreo}

Con base en la información de la Federación de Ganaderos de Sucre, Secretaría de Agricultura, ICA, UMATA, vacunadores, ganaderos, se determinó aproximadamente el número de fincas establecidas por corregimiento con sólo pasto angletón, de acuerdo con el Tabla 1.

Las fincas ganaderas seleccionadas fueron muestreadas y en cada una de ellas se llevó a cabo un muestreo aleatorio tomando de 20 a 25 submuestras por finca a una profundidad de 0 a $20 \mathrm{~cm}$; recolectando al tiempo suelos y raíces. Posteriormente estas submuestras se homogenizaron para conformar unas muestras de cada una de las fincas con un peso aproximado de $2 \mathrm{Kg}$, las cuales se depositaron en bolsas plásticas, rotuladas con el número de la finca, municipio y fecha de recolección. A cada finca a muestreada, se le diligenció un formulario encuesta. Las muestras tomadas de cada finca ganadera, se dividieron en dos porciones iguales de $1 \mathrm{Kg}$ para realización de análisis fisicoquímicos y microbiológicos respectivamente. 
Tabla 1. Número de fincas por corregimientos del municipio de Tolú, sembradas con el pasto angletón

\begin{tabular}{|c|c|c|}
\hline № & Corregimientos & № de Fincas con pasto angletón \\
\hline 1 & Cabecera Municipal & 14 \\
\hline 2 & Puerto Viejo & 12 \\
\hline 3 & Santa Lucía & 10 \\
\hline 4 & Límites Coveñas & 10 \\
\hline 5 & Nueva Era & 9 \\
\hline 6 & Pita En Medio & 9 \\
\hline 7 & Pita Abajo & 9 \\
\hline & Total & $\mathbf{7 3}$ \\
\hline
\end{tabular}

\section{Análisis microbiológico.}

El análisis microbiológico fue realizado en el laboratorio de microbiología de la Universidad de Sucre. Cada muestra de suelos provenientes de las diferentes fincas ganaderas fue tamizadas para retirar partes partículas gruesa (piedras, cascajos) y raíces. Una vez realizado el tamizado, se procedió al aislamiento de esporas, mediante la técnica propuesta por PEREZ (2003). De cada muestra procesada por la técnica anterior, se tomó dos mililitros de cada una de ellas, se depositó en cámara cuenta nemátodos y se realizó su conteo respectivo. Cada conteo se hizo tres veces por muestra, para obtener un estimativo del número total de esporas en 100 gramos de suelos por muestra.

Para determinar el porcentaje de colonización de HMA, se utilizaron raíces de aproximadamente un centímetro de longitud, se depositaron en frascos boca anchas estériles, se lavaron varias veces con agua y procedió a su respectiva tinción, utilizando la técnica propuesta por PEREZ (2003).

Las raíces coloreadas por la técnica anterior, en un número de 10 a 15 , se colocaron paralelamente sobre láminas, se cubrieron con cubreobjetos y se observaron al microscopio en objetivo de 40X, realizando el conteo en 100 campos ordenadamente. En cada campo se observó y se contó el número de campos negativo y positivo. Cuando el campo fue positivo, se determinó el tipo de estructura presente como: arbúsculos, vesículas, hifas y esporas. El resultado obtenido se multiplicó por 100 para expresar la colonización en porcentaje (PEREZ, 2003).

\section{Análisis estadístico}

Para establecer modelo matemático que permitieran predecir el comportamiento de las variables dependientes (porcentaje de colonización y número de esporas 
por $100 \mathrm{~g} /$ suelo) en función de las variables independientes (pH, MO, S, P, Ca, Mg, $\mathrm{K}, \mathrm{Na}, \mathrm{ClC}, \mathrm{Cu}, \mathrm{Fe}, \mathrm{Zn}, \mathrm{Mn}, \mathrm{Da}, \% \mathrm{H}, \mathrm{pH}$ del extracto, $\mathrm{CEE}, \mathrm{Ca}, \mathrm{Mg}, \mathrm{K}, \mathrm{Na}, \mathrm{HCO}_{3}$, $\mathrm{Cl}, \mathrm{SO}_{4}, \mathrm{PSI}, \mathrm{S} / 0_{00}$ ) producto de los resultados de análisis de caracterización y salinidad, se utilizaron técnicas de regresión lineal multivariado (LEBART et al. 1995). Inicialmente, se procedió a seleccionar las variables significativas para cada uno de los modelos, utilizando los procedimientos de regresión lineal multivariada de Stepwise, backward y forward y las pruebas t student (t valor) y $f$ valor. Finalmente se hizo el análisis de residuales para verificar la validez de cada uno de los modelos. Este análisis comprendió la realización de pruebas de media cero y pruebas de normalidad. Dentro de las pruebas de media cero se realizaron las pruebas $\mathrm{t}$, signo y rango signado. Las pruebas de normalidad utilizadas fueron la prueba de Shapiro-Wilks y la prueba de Kolmogorov-Smirnov. Los procedimientos estadísticos para el análisis de datos fueron ejecutados sobre el software SAS.

\section{Resultados}

En la Tabla 1 se registra los promedios de densidad de esporas para 100 gramos de suelo por fincas y por corregimientos encontrados en el presente estudio.

- Problemas de acidez, el problema de acidez no es notorio debido a la saturación de aluminio, comparada con la sumatoria del porcentaje de saturación de base.

- Problemas de Calcio (Ca), las fincas Mundo Viejo, la Victoria y El Paraíso poseen suelos donde predominan las sales de cloruro de calcio pero no presentan valores de $\mathrm{pH}$ alto.

En la Tabla 2, se registra los porcentajes promedios de colonización de HMA en raíces de pasto angletón por fincas y por corregimientos, los cuales fueron obtenidos a partir de tres repeticiones realizadas sobre las muestras.

Un total de 35 fincas ganaderas fueron muestreadas, las cuales de acuerdo a los resultados de los análisis físico-químicos y de salinidad presentaron las siguientes características y consideraciones generales. 
Tabla 1. Densidad de esporas promedio de tres repeticiones de HMA en fincas ganaderas del municipio de Tolú

\begin{tabular}{|c|c|c|c|c|c|}
\hline & \multirow{2}{*}{ Finca } & \multicolumn{4}{|c|}{ Esporas /100 g suelo } \\
\hline & & 1 & 2 & 3 & Promedio \\
\hline \multirow{7}{*}{ 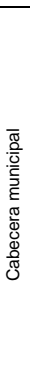 } & Pasatiempo & 768 & 719 & 625 & 704 \\
\hline & Santa Isabel & 745 & 646 & 710 & 700 \\
\hline & Macumbita & 832 & 632 & 625 & 696 \\
\hline & Manzanillo & 864 & 687 & 518 & 690 \\
\hline & Puerto Lupe & 702 & 622 & 459 & 594 \\
\hline & Puerto Cesar & 666 & 570 & 468 & 568 \\
\hline & Mora Palacio & 459 & 555 & 507 & 507 \\
\hline \multirow{4}{*}{ 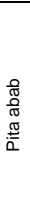 } & La Fortuna & 1.995 & 1.963 & 1.875 & 1944 \\
\hline & Santorini & 1.350 & 1.096 & 917 & 1121 \\
\hline & El ubérrimo & 900 & 896 & 700 & 832 \\
\hline & San Silvestre & 319 & 369 & 495 & 394 \\
\hline \multirow{3}{*}{ 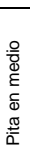 } & Mónaco & 808 & 658 & 588 & 685 \\
\hline & Macayepo 5 & 495 & 424 & 350 & 423 \\
\hline & Macayepo 6 & 408 & 350 & 336 & 365 \\
\hline \multirow{5}{*}{ 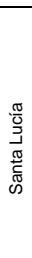 } & Galindo & 1.998 & 1.725 & 1.328 & 1684 \\
\hline & Ocala & 1.530 & 1.380 & 1.205 & 1372 \\
\hline & Puerta Azul & 1.204 & 906 & 745 & 952 \\
\hline & El Delirio & 675 & 579 & 500 & 585 \\
\hline & La Victoria & 476 & 398 & 351 & 408 \\
\hline \multirow{4}{*}{ 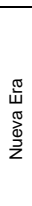 } & La Pecadora & 756 & 762 & 600 & 706 \\
\hline & El Tigre & 750 & 630 & 496 & 625 \\
\hline & Palma Sola & 576 & 574 & 482 & 544 \\
\hline & Filadelfia & 472 & 504 & 396 & 457 \\
\hline \multirow{6}{*}{$\begin{array}{l}\frac{0}{0} \\
\stackrel{2}{2} \\
0 \\
\frac{2}{0} \\
0 \\
0\end{array}$} & Caño Salao & 2.224 & 1.827 & 1.698 & 1916 \\
\hline & Villa Julia & 2.112 & 1.806 & 1.578 & 1832 \\
\hline & La Linda & 1.759 & 2.161 & 1.269 & 1730 \\
\hline & Marenco & 1.200 & 1.260 & 1.000 & 1153 \\
\hline & Puerto Arturo & 442 & 450 & 370 & 421 \\
\hline & Paraíso & 416 & 350 & 294 & 353 \\
\hline \multirow{5}{*}{ 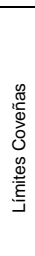 } & San Vicente & 2.420 & 2.088 & 1.904 & 2137 \\
\hline & Alicante & 1.938 & 1.632 & 1.545 & 1705 \\
\hline & Petalaca & 1.528 & 1.484 & 1.182 & 1398 \\
\hline & Buenos Aires & 1.225 & 1.139 & 1.050 & 1138 \\
\hline & Limonar & 765 & 870 & 761 & 799 \\
\hline
\end{tabular}


- Problemas de salinidad y sodificación, en la finca Petalaca se encontró que por sus características el suelo se puede clasificar como sulfatado ácido sódico, con alto contenido de azufre, este elemento se oxida originando ácido sulfúrico en condiciones de aireación y en condiciones de alta humedad los reduce. La finca Pasatiempo presenta una relación calcio magnesio invertida, con porcentaje de saturación de magnesio mayor del $40 \%$, encontrándose sales como cloruros y sulfatos de magnesio de manera similar que en las fincas Ubérrimo, Ocala, El Tigre, Caño Salao y Villa Julia. La presencia de altos porcentajes de saturación de magnesio (>30\%) es síntoma de la magnesificación de los suelos, la cual es ayudada por la existencia de las arcillas presentes. De acuerdo a estas características se clasifican como suelos magnésicos. Estos resultados son similares a lo planteado por García (1994), quien ha reportado que el efecto desfloculante del magnesio es parecido al del sodio, por lo que puede ser notorio la perdida de la estructura y las condiciones masivas de los suelos en un ambiente hidromórfico, de igual manera se reporta para estos suelos unas altas concentraciones fósforo la cual puede estar asociada a la materia orgánica presente. En Puerto Cesar, Puerto Arturo, Macumbita y Caño Salao se encontraron suelos con características de salino con posibles problemas de sodio, debido al tipo de arcilla (2:1) de estos suelos.

- Problemas de Materia Orgánica (MO), para la mayoría de los suelos de las fincas está en un rango de normal-medio (2\% - 4\%). Sin embargo; Manzanillo, Petalaca, Buenos Aires y Villa Julia, presentan valores muy altos que no son concordantes con las condiciones ecológicas de la zona. Esto probablemente es debido a las condiciones no favorables para la actividad de los organismos, tales como humedad, saturación, aireación.

Tabla 2. Porcentaje de colonización promedio de HMA en fincas ganaderas del municipio de Tolú

\begin{tabular}{|c|c|c|c|c|c|}
\hline & \multirow[t]{2}{*}{ Finca } & \multicolumn{4}{|c|}{ \% Colonización de raíces } \\
\hline & & 1 & 2 & 3 & Promedio \\
\hline \multirow{6}{*}{ 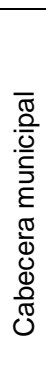 } & Pasatiempo & 22 & 23 & 21 & 22 \\
\hline & Santa Isabel & 14 & 20 & 25 & 20 \\
\hline & Macumbita & 52 & 49 & 55 & 52 \\
\hline & Manzanillo & 38 & 43 & 41 & 41 \\
\hline & Puerto Lupe & 61 & 68 & 64 & 64 \\
\hline & Puerto Cesar & 27 & 29 & 25 & 27 \\
\hline
\end{tabular}




\begin{tabular}{|c|c|c|c|c|c|}
\hline & Mora Palacio & 60 & 53 & 46 & 53 \\
\hline \multirow{4}{*}{ 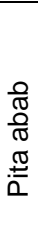 } & La Fortuna & 57 & 64 & 61 & 61 \\
\hline & Santorini & 30 & 23 & 16 & 23 \\
\hline & El ubérrimo & 45 & 39 & 34 & 19 \\
\hline & San Silvestre & 43 & 52 & 34 & 43 \\
\hline \multirow{4}{*}{ 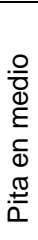 } & Mónaco & 35 & 19 & 27 & 27 \\
\hline & Mundo Viejo & 21 & 11 & 30 & 21 \\
\hline & Macayepo 5 & 54 & 48 & 51 & 51 \\
\hline & Macayepo 6 & 71 & 65 & 60 & 65 \\
\hline \multirow{5}{*}{ 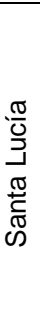 } & Galindo & 28 & 35 & 20 & 28 \\
\hline & Ocala & 28 & 16 & 22 & 22 \\
\hline & Puerta Azul & 53 & 44 & 36 & 44 \\
\hline & El Delirio & 38 & 25 & 51 & 38 \\
\hline & La Victoria & 50 & 55 & 60 & 55 \\
\hline \multirow{4}{*}{ 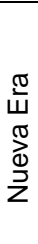 } & La Pecadora & 41 & 36 & 47 & 41 \\
\hline & El Tigre & 38 & 46 & 42 & 42 \\
\hline & Palma Sola & 46 & 59 & 53 & 53 \\
\hline & Filadelfia & 71 & 76 & 80 & 76 \\
\hline \multirow{6}{*}{ 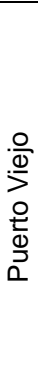 } & Caño Salao & 68 & 61 & 75 & 68 \\
\hline & Villa Julia & 28 & 44 & 49 & 44 \\
\hline & La Linda & 26 & 40 & 33 & 33 \\
\hline & Marenco & 38 & 31 & 35 & 35 \\
\hline & Puerto Arturo & 43 & 46 & 40 & 43 \\
\hline & Paraíso & 48 & 52 & 44 & 48 \\
\hline \multirow{5}{*}{ 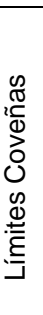 } & San Vicente & 30 & 27 & 24 & 27 \\
\hline & Alicante & 36 & 40 & 31 & 36 \\
\hline & Petalaca & 37 & 43 & 40 & 40 \\
\hline & Buenos Aires & 28 & 30 & 26 & 28 \\
\hline & Limonar & 41 & 39 & 36 & 39 \\
\hline
\end{tabular}

- Problemas de fósforo $(P)$, los valores altos a muy altos de fósforo en la mayoría de las fincas son debidos a la presencia de fosfato de alta solubilidad extraídos por el método de Bray II (ácido). La finca Limonar es la única que presenta suelos con contenido medio de este elemento. 
- Problemas de reacción del suelo $(\mathrm{pH})$, las fincas Pasatiempo, San Silvestre, el Delirio, Caño Salao, Marenco y Petalaca presentan suelos muy ácidos pero sin problemas de aluminio intercambiable, en el resto de fincas se encuentran suelos moderadamente ácidos a ligeramente alcalinos. El problema de acidez de estos suelos es debido a la alta presencia de sulfato los cuales se han originado a partir de los procesos acelerados de la descomposición de la materia orgánica, ya que la posición geomorfológica de la planicie en las cuales se encuentran estas fincas produce una ganancia o acumulación de materiales orgánicos en condiciones hidromórficas.

- Problemas de azufre (S), a excepción de las fincas de Puerto Arturo, Macumbita y Petalaca que presentan valores muy altos y extremadamente altos de azufre, los suelos de las otras fincas este elemento se encuentran en un rango normal.

- Problemas de bases intercambiables, el contenido se encuentra normal para la mayoría de las fincas, al igual que el porcentaje de saturación, el problema radica en la relación calcio magnesio que es muy estrecha exceptuando las fincas Mundo Viejo, La Victoria y Paraíso que tienen una relación amplia. Las fincas Pasatiempo, El Uberrimo, Ocala, El Tigre, Caño Salao y Villa Julia tienen relación invertida correspondiendo a suelos magnésicos.

- Problemas de extractos de saturación, la mayoría de las fincas se encuentran en condiciones normales exceptuando las fincas Puerto Cesar, la Victoria, Villa Julia y Puerto Arturo. Las fincas Alicante y Petalaca muestran altos contenidos de extracto de saturación pero en Petalaca los contenidos de calcio y magnesio están en relación inversa.

- Problemas elementos menores (Fe, $\mathrm{Mn}, \mathrm{Cu}, \mathrm{Zn})$, el Hierro se encuentra en valores excesivo en las fincas Macumbita, Caño Salao y deficiente en la finca Santa Isabel y Limonar. El Manganeso es excesivo en la finca Macumbita, Caño Salo, Villa Julia y Marenco. El antagonismo del hierro con el manganeso es debido a que la relación hierro-manganeso en algunas de las fincas es menor de 1.

\section{Determinación de modelo matemático}

El análisis de regresión lineal por la técnica de Backward permitió obtener el modelo matemático que relaciona la densidad poblacional de esporas (número de esporas por $100 \mathrm{~g}$ de suelo), de HMA en función de parámetros físico-químico encontrado en los suelos de las diferentes fincas ganaderas en el momento del presente estudio (Fig.1). 


$$
\text { Densidad poblacional }=83.07_{\mathrm{Mg}}+715.67_{\mathrm{Ha}}-260.63_{\mathrm{Cu}}-7.60_{\mathrm{Mn}}+428.57_{\mathrm{Da}}
$$

Figura 1. Modelo matemático para esporulación de HMA en función de parámetros físicos-químicos de suelo de fincas ganaderas del municipio de Tolú. Da: densidad aparente.

Los modelos matemáticos construidos para densidad poblacional de esporas de HMA en función de parámetros físicos-químicos, indican que la abundancia de esporas de HMA en los suelos está directamente relacionada a la concentración de los elementos intercambiables de magnesio $(\mathrm{Mg})$, sodio $(\mathrm{Na})$ y de los valores de densidad aparente ( $\mathrm{Da}$ ) e inversamente relacionada a los valores de Cobre (Cu) y Manganeso (Mn).

Además del anterior modelo, fue establecido otro modelo que relaciona el número de esporas por 100 gramos de suelo (densidad poblacional), en función de las variables independientes $\mathrm{pH}$ del extracto, $\mathrm{CEE}, \mathrm{Ca}, \mathrm{Mg}, \mathrm{K}, \mathrm{Na}, \mathrm{HCO}_{3}, \mathrm{Cl}, \mathrm{SO}_{4}$, $\mathrm{PSI}, \mathrm{S} /{ }_{00}$, las cuales están directamente relacionadas a la presencia de salinidad de los suelos, mediante análisis de regresión lineal multivariado de Stepwise (Fig. 2).

$$
\text { Densidad poblacional }=135.32_{\mathrm{pH}}-3662.8_{\mathrm{CEE}}+109.04_{\mathrm{Mg}}+218.29_{\mathrm{Na}}+294.80_{\mathrm{S} 04}+91.97_{\mathrm{PSI}}+1668.55_{\mathrm{S} N 00}
$$

Figura 2. Modelo matemático para esporulación de HMA en función de parámetros físicos-químicos, que relacionan la salinidad de los suelos de fincas ganaderas del municipio de Tolú. CEE: conductibilidad eléctrica, PSI: \% de sodio intercambiable, s/00: Salinidad en partes por mil.

Los modelos matemáticos construidos para densidad poblacional de esporas de HMA en función de salinidad, indican que la salinidad interfiere directamente con el número de espora de esporas de HMA en los suelos en presencia de parámetros como $\mathrm{pH}, \mathrm{SO} 4$, magnesio $(\mathrm{Mg})$, sodio $(\mathrm{Na})$, porcentaje de sodio intercambiable (PSI) y el porcentaje de sodio en partes por mil $(\mathrm{S} / 00)$ e inversamente relacionada a la conductividad eléctrica (CEE).

Después de haber realizado los modelos que relacionan el número de esporas por $100 \mathrm{~g}$, en función de parámetros físicos-químicos y de salinidad, se realizaron 
procedimientos similares para construir el modelo matemático que definen la colonización de HMA, en función de parámetros físicos-químicos, mediante modelo de regresión simple (fig. 3) y de salinidad, por medio de regresión lineal mediante el método de Backward (Fig. 3).

$$
\text { \%colonización }=-0.42_{\mathrm{Ca}}-1.01_{\mathrm{Mg}}+8.53_{\mathrm{K}}+8.154_{\mathrm{Ha}}+3.7_{\mathrm{Zn}}+1.02_{\% \mathrm{H}}
$$

Figura 3. Modelo matemático para \% de colonización de HMA en raíces de pasto angletón en función de parámetros físicos-químicos de suelos de fincas ganaderas del municipio de Tolú.

El modelo anterior señala que la colonización de HMA en raíces de angletón está directamente relacionada a los valores de los elementos intercambiables de potasio $(\mathrm{K})$ y sodio $(\mathrm{Na})$, de zinc $(\mathrm{Zn})$ y del porcentaje de hidrogeno en los suelos e inversa a los valores de los elementos de Ca y Mg intercambiable.

$$
\text { \%colonización }=30.19+5.31_{\mathrm{Ca}}-5.10_{\mathrm{Mg}}-7.26_{\mathrm{HCO} 3}+6.139_{\mathrm{PSI}}
$$

Figura 4. Modelo matemático para \% de colonización de HMA en raíces de pasto angletón en función de parámetros físicos-químicos, asociados a salinidad de suelos de fincas ganaderas del municipio de Tolú

El modelo matemático de la Fig. 4 muestra que la salinidad interfiere con el porcentaje de colonización de HMA en raíces de angletón en presencia del elemento calcio intercambiable y de los valores de PSI e inversamente a los valores del elemento Magnesio intercambiables y de ácido carbónico $\left(\mathrm{HCO}_{3}\right)$.

\section{Discusión}

A nivel mundial y nacional, son escasas las investigaciones sobre integración y evaluación del efecto de parámetros físicos, químicos y biológicos con relación a la calidad del ecosistema suelo. Los estudios que existen son pocos conclusivos y de difícil interpretación, dada la gran complejidad del sistema en estudio en donde se evalúan varias características, generando un número mayor de datos, lo que proporcionalmente dificulta su interpretación y análisis (TOTOLA y CHAER, 2002). La presencia y el establecimiento de los HMA en condiciones de campo están determinado por diversos factores tales como: físico-químicas del suelo $(\mathrm{pH}$, contenido de fósforo, temperatura, aireación, textura y contenido de materia 
orgánica), condiciones climáticas (intensidad y duración de la luz, temperaturas, humedad, épocas de lluvias y épocas secas) y por las prácticas agronómicas (preparación del terreno, aplicación de pesticidas y prácticas culturales) (SANCHEZ, 1999).

PEREZ y FUENTES (2009), encontraron que en el proceso de modelación logística de la densidad poblacional de HMA en función de parámetros físicosquímicos; los factores que mejor explican el fenómeno de la esporulación de hongos formadores de micorrizas asociados en rizosfera de pasto colosuana fueron el $\mathrm{pH}$ y el Potasio. Diversos estudios in situ han demostrado que entre los factores físicos-químico que más influyen en el desarrollo de las HMA se encuentra el contenido de $\mathrm{pH}$ en los suelo; sin embargo, se ha comprobado que los hongos formadores de micorrizas arbusculares tienen amplia capacidad de adaptación a condiciones de $\mathrm{pH}$, éstos se han registrado desde valores de 2.7 a 9.2. Se encuentran diferencias entre especies y ecotipos en cuanto a su capacidad para colonizar en función del pH (SANCHEZ, 1999).

MOSSE, (1991), señala que en los suelos áridos y semiáridos el exceso de sales solubles es un problema especial, debido a que la presencia de salinidad causa desbalance nutricional para las plantas. Un exceso de cloro puede interferir en la absorción de nitratos y fosfatos, e igualmente una alta concentración de sodio puede afectar la adquisición de calcio y magnesio. Se ha demostrado que los HMA pueden conferir cierta tolerancia a las plantas frente a condiciones de estrés y por esta razón se cree que estos hongos pueden aliviar algunos efectos negativos de la salinidad. En otro estudio realizado por MERGULHAO et al., (2001), en Brasil con el pasto Brachiaria decumbens, inoculado con la especie de HMA Glomus etunicatum, en suelos con diferentes niveles de cloruro de sodio encontró que el porcentaje de infecciones en raíces en esta especie de pasto y el número de esporas en el suelo no fue afectado por incrementos en los niveles de cloruro de sodio aplicados al suelo. Sin embargo MCMILLEN et al., (1999), en estudio realizado en Australia, sobre el efecto del cloruro de sodio sobre en la colonización de Gigaspora decipiens dentro de las raíces de Trifolium resupinatum encontró que incrementos en la concentración de cloruro de sodio en el suelo inhibe la distribución de la colonización después de iniciada, debido a que la concentración de este compuesto inhibe el crecimiento de la hifa e influye en el suministro de carbohidratos desde la planta hacia el hongo.

En la agricultura sustentable, el uso de HMA tiene un potencial biotecnológico debido a que facilita la disponibilidad de nutrientes para las plantas. Las plantas micorrizadas in situ poseen una ventaja importante con respecto a las no micorrizadas. Sin embargo, el conocimiento sobre las interacciones entre las 
condiciones edáficas, la ecología de los HMA nativos y el establecimiento de simbiosis entre las plantas y estos microorganismos es limitado. Por tal motivo, el análisis de poblaciones de HMA nativos en condiciones edafoclimáticas de agroecosistemas de pasturas tropicales, se convierte en nuevas fronteras de estudio para su uso, manejo y conservación eficiente en la agricultura sostenible de países en vías de desarrollo.

\section{Agradecimientos}

Los autores expresan sus agradecimientos a la UMATA Santiago de Tolú y asociaciones ganaderas del departamento de Sucre.

\section{Referencias}

AGUILERA, M.M. 2005. Documento de trabajo sobre economía regional. Economía Regional: La Economía del Departamento de Sucre: Ganadería y Sector Público. Ediciones Banco Ganadero, Sincelejo.

BAREA, J. 1999. Importancia de las micorrizas arbusculares en el establecimiento y protección de las plantas en suelos degradados. Phytoma 111:29-56.

INSTITUTO GEOGRÁFICO AGUSTÍN CODAZZI. 1998. Estudio general de suelos y zonificación de tierras, Departamento de Sucre. Instituto Geográfico Agustín Codazzi, Sincelejo.

JEFFRIES, P.; GIANINAZZI, S.; PEROTTO, S., TURNAU, K., BAREA. J.M. 2003. The contribution of arbuscular mycorrhizal fungi in sustainable maintenance of plant health and soil fertility. Biology and Fertility of Soils 37(1):1-16. http://www.springerlink.com/content/0wegr2nj6f16m7cl/fulltext.pdf

KENNEDY, A.C.; SMITH, K.L. 1995. Soil microbial diversity and the sustaninbability of agricultural soils. Plant and soil 170(1):75-86. http://www.springerlink.com/content/x60450v25036102j/fulltext.pdf.

LEBART, L.; MORINEAU A.; PIRON, M. 1995. Statisitique exploratoire multidimensionnelle. ediciones Dunod, Paris.

McMILLEN, B.G.; JUNIPER, S.; ABBOT, L.K. 1998. Inhibition of hyphal growth of a vesicular arbuscular mycorrhizal cantaining sodium chloride of infection from fungus in soil limits the spread spores. Soil Biology and Biochemestry 30(13):16391646.

MOSSE, B. 1991. Advances in the study of VAM. Annual rev. Phytopathology 11: 171-196. 
MERGULHAO, A.C.E.S; BURUTY, H.A; TABOSA, J.N; FIGUEIREDO, M.V.B; MAIA, L.C. 2001. Salt stress response of Brachiaria plants with and without inoculation of arbuscular mycorrhizal fungi. Agrochimica 45(1-2):24-31.

PÉREZ, C.A. 2003. Eficiencia de hongos formadores de micorrizas arbusculares (H.M.A.) nativos, asociados a la producción de forraje en la especie de pasto colosuana (Bothriochloa pertusa (L) A. camus) en el municipio de corozal; departamento de Sucre. Tesis de Maestría. Universidad de los Andes, Facultad de Ciencias, Departamento de Ciencias Biológicas. Bogotá, Colombia.

PEREZ, A.; FUENTES, J. 2009. Regresión logística en la evaluación de la esporulación de micorrizas en pasto Bothriochloa pertusa (L) A. Camus. Rev. Colombiana cienc. Anim 1(1):1-18. http://www.recia.edu.co/documentosrecia/4 Micorrizas Perez\&Fuentes.pdf

PLAN DE ORDENAMIENTO TERRITORIAL. 2000. Municipio Santiago de Tolú, componente rural. Consultor: Hernández \& Cía. Sincelejo.

SÁNCHEZ, M. 1999. Endomicorrizas en agroecosistemas colombianos. Ed. Universidad Nacional de Colombia, sede Palmira, Bogotá.

TOTOLA, M.; CHAER, G. 2002. Microrganismos e processos microbiológicos como indicadores da qualidade dos solos. Rev. Bras. Cienc. Solo 2:249-259.

VILORIA, H.J. 2002. Documento de trabajo sobre economía regional: La ganadería bovina en las llanuras del Caribe Colombiano. Banco de la República, Cartagena de Indias. 